\title{
A Simple Construction of 16-QAM Codewords with Low PMEPR for OFDM Signals
}

\author{
Wen Chen \\ Department of Electronics Engineering \\ Shanghai Jiaotong University \\ 800 Dongchuan Road, Minhang, Shanghai, China 200240 \\ Email: wenchen@sjtu.edu.cn
}

\author{
Chintha Tellambura \\ Department of Electrical and Computer Engineering \\ University of Alberta \\ Edmonton, AB, Canada, T6G 2V4 \\ Email: chintha@ece.ualberta.ca
}

\begin{abstract}
Golay sequences have been introduced to construct 16-QAM (quaternary amplitude modulation) code for the orthogonal frequency division multiplexing (OFDM), reducing the peak-to-mean envelope power ratio (PMEPR). As an alternative way to construct Golay sequences, the construction of 16QAM using Rudin-Shapiro polynomials (RSP) was also reported recently in several literatures. In this paper, we develop a simple effcient construction of 16-QAM using generalized RSP, which provides high code rate and large Hamming distance while tightly controlling the PMEPR.
\end{abstract}

\section{INTRODUCTION}

Orthogonal frequency division multiplexing (OFDM) eliminates the need for complex equalizers in wide-band fading channels, while efficient hardware implementations can be realized using the fast Fourier transform (FFT). However, a major drawback of OFDM signals is the high peak-tomean envelope power ratio (PMEPR) of the uncoded OFDM signal. Some popular PMEPR reduction techniques include signal distortion techniques [1], [2], coding [3], [4], [5], [6], multiple signal representation [7], [8], [9], [10], modified signal constellation [11], pilot tone methods [12] and others.

An idea introduced in [13] and developed in [14] is to use the Golay sequences [15] to encode OFDM signals with a PMEPR of at most 2. These sequences have been employed as pilot sequences by European Telecommunications Standards Institute (ETSI) Broad Radio Acess Networks (BRAN). Recently Davis and Jedwab [3] made an attractive theoretical advance on this work and observed that the $2^{h}$-ary Golay sequences of length $2^{m}$ can be obtained from certain second order cosets of the classical first order Reed-Muller code. As a consequence of this intrinsic observation, Davis and Jedwab [4] were able to obtain $(m ! / 2) 2^{h(m+1)}$ codewords (DJ-code) for the phase shift keying (PSK) OFDM signals of $2^{m}$ carriers with good error-correcting capabilities, efficient encoding and decoding, and a PMEPR of at most 2. Futher investigation on DJ-code has been done in [6], which employs Golay set [16] to increase the code rate by relaxing the PMEPR.

Since quadrature amplitude modulation (QAM) sequences are widely used in OFDM, RöBing and Tarokh[17] has studied the Golay sequences for 16-QAM OFDM signals. By decomposing a 16-QAM symbol uniquely into a pair of quaternary
PSK (QPSK) symbols, they construct $\left[(m ! / 2) 4^{(m+1)}\right]^{2} 16$ QAM OFDM signals of length $2^{m}$ starting from Golay QPSK sequences and provide bound 3.6 on their PMEPR. More detailed construction of 16-QAM OFDM signals using Golay sequneces has been recently reported in [18].

As a simple way to construct Golay sequences, the RudinShapiro polynomials (RSP) [19] have been studied in [20], [21], [22]. They obtain $2^{h(m+1)}$ codewords for $2^{h}$-ary OFDM signals of $2^{m}$ carriers. Although the code rate of RSP based construction is lower than that of DJ-code, this simple construction matches with some efficient decoding scheme [23]. Further study to increase the code rate has been reported in [20]. Recently, RSP has also been used to construct 16QAM code in [24], which obtains $m 4^{m+3}-256(m-1)$ codewords of length $2^{m}$. In this paper, we develop a simple way to design the 16-QAM OFDM signals using the generalized RSP [20]. We can obtain $4^{3(2 k)+2}$ codewords of length $2^{2 k}$ and provide bound 7.2 for PMEPR. Let $m=2 k$, we actually construct $4^{3 m+2}$ codewords of length $2^{m}$, which is more than those presented in [18], [24] for modurately large carriers.

\section{PRElimilaries}

Let $j$ be the imaginary unit, i.e., $j^{2}=-1$. For an $M$-ary phase modulation OFDM, let $\xi^{\mathbb{Z}_{M}}=\left\{\xi^{k}: k \in \mathbb{Z}_{M}\right\}$, where $\xi=\exp (2 \pi j / M)$ and $\mathbb{Z}_{M}=\{0, \cdots, M-1\}$.

\section{A. OFDM signals, instantaneous power and PMEPR}

For a codeword $c=\left(c_{0}, \ldots, c_{n-1}\right)$ with $c_{\ell} \in \xi^{\mathbb{Z}_{M}}$, the $n$ subcarrier complex baseband OFDM signal can be mathematically simplified as

$$
s_{c}(z):=\sum_{\ell=0}^{n-1} c_{\ell} z^{\ell},
$$

where $z=e^{j 2 \pi t}$. The instantaneous power of the complex envelope $s_{c}(z)$ is defined by

$$
P_{c}(z):=\left|s_{c}(z)\right|^{2} .
$$

The peak-to-mean envelope power ratio (PMEPR) of the codeword $c$ is defined as

$$
\operatorname{PMEPR}(c):=\frac{1}{n} \sup _{|z|=1} P_{c}(z) .
$$




\section{B. Aperiodic auto-correlation and Golay sequences}

For a sequence $a \in \mathbb{C}^{n}$, the aperiodic auto-correlation function $R_{a}(\cdot)$ is defined by

$$
R_{a}(\ell)=\left\{\begin{array}{cl}
\sum_{k=0}^{n-\ell-1} a_{k+\ell} \bar{a}_{k}, & \ell=0,1, \cdots, n-1, \\
0, & \text { otherwise },
\end{array}\right.
$$

where $\bar{a}_{k}$ is the complex conjugate of $a_{k}$.

Golay sequences were originally introduced to deal with the optical problem of multislit spectrometry. Golay also predicted that it will have possible application in communication engineering [15], which was recently fulfilled by the works done in [3], [4], [5], [6]. The original Golay sequences are defined only for the binary sequences [15]. However, it can be easily extended to the $M$-ary sequences as recently done in [4], [5], [6]

A pair of sequences $a$ and $b$ of length $n$ are said to form a Golay pair if

$$
P_{a}(z)+P_{b}(z)=2 n .
$$

The sequences $a$ and $b$ are called Golay sequences.

Obviously, $\operatorname{PMEPR}(a) \leq 2$ if $a$ is a Golay sequence, which tightly controls the PMEPR of the underlying OFDM signal $s_{a}(z)$ by 2 . This is a big advantage of using Golay sequences to reduce PMEPR for OFDM signals. Using the aperiodic auto-correlation function, the instantaneous power $P_{a}(z)$ of the sequence $a$ can be represented as

$$
P_{a}(z)=R_{a}(0)+\sum_{\ell=1}^{n-1}\left[R_{a}(\ell) z^{\ell}+\bar{R}_{a}(\ell) z^{-\ell}\right] .
$$

Therefore, equation (4) is equivalent to

$$
R_{a}(\ell)+R_{b}(\ell)=2 n \delta(\ell),
$$

where $\delta(\ell)$ is Dirac sequence, which takes the value 1 at 0 , and takes the value 0 elsewhere.

\section{Rudin-Shapiro polynomials}

Besides those introduced in the introduction, the early application of Rudin-Shapiro polynomials (RSP) [19] to constructing encoding and decoding schemes for OFDM can be found in [25].

For a $k \geq 0$, an $\mathrm{RSP}$ pair $(A(z), B(z))$ is recursively defined as

$$
\left\{\begin{array}{l}
A_{k+1}(z)=A_{k}(z)+\xi_{k} z^{2^{k}} B_{k}(z), \\
B_{k+1}(z)=A_{k}(z)-\xi_{k} z^{2^{k}} B_{k}(z),
\end{array}\right.
$$

where $A_{0}(z)=B_{0}(z)=1$ and $\xi_{k}$ is any element in $\xi^{\mathbb{Z}_{M}}$.

Formula (5) recursively produces the polynomials $A_{k}(z)$ and $B_{k}(z)$ of degree $2^{k}-1$ for any $k>0$. In general, for $n=2^{m}$, let the sequences $a$ and $b$ be, respectively, the coefficients of the polynomials $A_{m}(z)$ and $B_{m}(z)$. The $2^{m}$ subcarrier OFDM signals are $s_{a}(z)=A_{m}(z)$ and $s_{b}(z)=$ $B_{m}(z)$. For example, for $m=3$, we have $n=8$ and the codewords

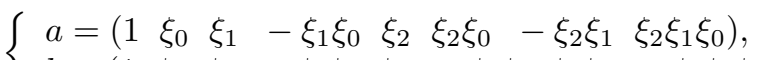

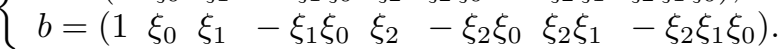

From (5), it is clear that

$P_{a}(z)+P_{b}(z)=\left|s_{a}(z)\right|^{2}+\left|s_{b}(z)\right|^{2}=\left|A_{m}(z)\right|^{2}+\left|B_{m}(z)\right|^{2}$ Noting $\left|A_{m}(z)\right|^{2}+\left|B_{m}(z)\right|^{2}=2\left[\left|A_{m-1}(z)\right|^{2}+\left|B_{m-1}(z)\right|^{2}\right]$ and repeating the process, we have

$$
P_{a}(z)+P_{b}(z)=2^{m}\left[\left|A_{0}(z)\right|^{2}+\left|B_{0}(z)\right|^{2}\right]=2 n .
$$

This shows that $a$ and $b$ form a Golay pair. Therefore, RSP pair constitute a subset of Golay pair. Hence the PMEPR of an RSP is at most 2 .

By the recursive formula (5), one can construct $M^{m}$ Golay sequences. Since for any $\eta \in \xi^{\mathbb{Z}_{M}}, \eta a$ is also a Golay sequence if $a$ is a Golay sequence, one can construct $M^{m+1}$ Golay sequences by RSP.

\section{NEW CONSTRUCTION OF 16-QAM CODEWORDS}

Our construction of 16-QAM is based on the generalized RSP and the decomposition of 16-QAM symbols. In the following, we will firstly introduce the generalized RSP, then construct the 16-QAM codewords using generalized RSP.

\section{A. Generalized RSP}

We now introduce the generalized RSP, for which we write the formula (5) in the matrix form. Let

$$
\mathbf{A}_{k}^{2}(z)=\left(\begin{array}{c}
A_{k}(z) \\
B_{k}(z)
\end{array}\right), \mathbf{B}_{k}^{2}(z)=\left(\begin{array}{c}
A_{k}(z) \\
z^{2^{k}} B_{k}(z)
\end{array}\right),
$$

and

$$
\mathbf{T}_{k}^{2}=\left(\begin{array}{cc}
1 & \xi_{k} \\
1 & -\xi_{k}
\end{array}\right) .
$$

Then one can rewrite formula (5) in the matrix form

$$
\mathbf{A}_{k+1}^{2}(z)=\mathbf{T}_{k}^{2} \mathbf{B}_{k}^{2}(z)
$$

This immediately suggests an extension of RSP. Let $\theta=$ $\exp (j 2 \pi / N)$. Extend $\mathbf{A}_{k}^{2}(z), \mathbf{B}_{k}^{2}(z)$ and $\mathbf{T}_{k}^{2}$ respectively to $\mathbf{A}_{k}^{N}(z), \mathbf{B}_{k}^{N}(z)$ and $\mathbf{T}_{k}^{N}$ as

$$
\begin{gathered}
\mathbf{A}_{k}^{N}(z)=\left(\begin{array}{c}
A_{k+1}^{0}(z) \\
A_{k+1}^{1}(z) \\
\vdots \\
A_{k+1}^{N-1}(z)
\end{array}\right), \mathbf{B}_{k}^{N}(z)=\left(\begin{array}{c}
A_{k}^{0}(z) \\
z^{N^{k}} A_{k}^{1}(z) \\
\vdots \\
z^{(N-1) N^{k}} A_{k}^{N-1}(z)
\end{array}\right), \\
\mathbf{T}_{k}^{N}=\left(\begin{array}{ccccc}
1 & \xi_{k}^{1} & \xi_{k}^{2} & \cdots & \xi_{k}^{N-1} \\
1 & \theta \xi_{k}^{1} & \theta^{2} \xi_{k}^{2} & \cdots & \theta^{N-1} \xi_{k}^{N-1} \\
\vdots & \vdots & \vdots & & \vdots \\
1 & \theta^{N-1} \xi_{k}^{1} & \theta^{2(N-1)} \xi_{k}^{2} & \cdots & \theta^{(N-1)(N-1)} \xi_{k}^{N-1}
\end{array}\right),
\end{gathered}
$$

where $A_{0}^{0}=\cdots=A_{0}^{N-1}=1$ and $\xi_{k}^{1}, \ldots, \xi_{k}^{N-1}$ are any symbols taken from the constellation $\xi^{\mathbb{Z}_{M}}$. Naturally, the generalized RSP vector is iteratively defined by the formula

$$
\mathbf{A}_{k}^{N}(z)=\mathbf{T}_{k}^{N} \mathbf{B}_{k}^{N}(z)
$$

Each polynomial entry in $\mathbf{A}_{k}^{N}(z)$ is called a generalied $R S P$. Obviously, the generalized RSP degenerates to the ordinary RSP if $N=2$. In this paper, we are interested in the case $N>2$. 


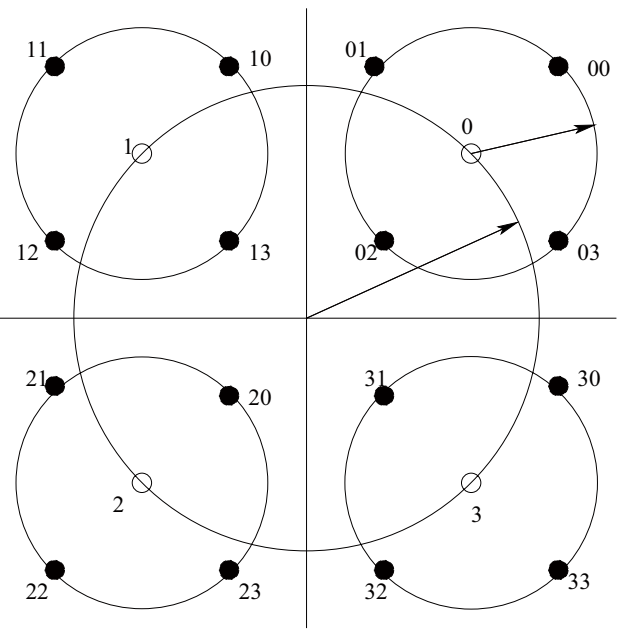

Fig. 1. Construction of 16-QAM from two QPSK.

\section{B. PMEPR of 16-QAM sequences by generalized RSP}

For a QPSK sequence $Q$ constructed in the last subsection, we can write it as

$$
Q=\alpha e^{j \pi / 4} U+\beta e^{j \pi / 4} V
$$

where $U=\left(\eta_{0}, \ldots, \eta_{n-1}\right)$ and $V=\left(\zeta_{0}, \ldots, \zeta_{n-1}\right)$ are the two QPSK sequences from generalized RSP (see Fig. 1).

Using the PMEPR of generalized RSP, one can obtain the PMEPR of 16-QAM sequences, which is summarized in the following theorem.

Theorem 1: For the 16-QAM sequence $Q$ of length $4^{k}$, constructed in the last subsection, the PMEPR is at most 7.2. Proof: Let $Z=\left(1, z, \ldots, z^{n-1}\right)^{T}$. Then the 16-QAM OFDM signal is $s_{Q}=Q Z$. Since $Q=\alpha e^{j \pi / 4} U+\beta e^{j \pi / 4} V$, we have

$$
s_{Q}(z)=\alpha e^{j \pi / 4} U Z+\beta e^{j \pi / 4} V Z .
$$

If we can show $\operatorname{PMEPR}(U) \leq B$ and $\operatorname{PMEPR}(V) \leq B$, then it is easy to see

$$
\left|s_{Q}(z)\right|^{2} \leq(\alpha+\beta)^{2} B n=\frac{9 B n}{5} .
$$

In order to show $\operatorname{PMEPR}(Q) \leq 7.2$, we only need to show $B=4$. In the following, we will verify this result.

Since $U$ and $V$ are two QPSK sequences constructed by generalized RSP. Without loss of generality, we can just investigate $U$. The derived result can be directly applied to $V$. Assume $U^{1} Z, U^{2} Z, U^{3} Z$ together with $U^{0} Z(=U Z)$ form the generalized RSP vector. From (6), we have

$$
\begin{aligned}
\sum_{\ell=0}^{3}\left|s_{U^{\ell}}(z)\right|^{2} & =\sum_{\ell=0}^{3}\left|A_{m}^{\ell}(z)\right|^{2} \\
& =\left(\mathbf{A}_{m}^{4}\right)^{\top} \cdot \overline{\mathbf{A}_{m}^{4}},
\end{aligned}
$$

where $\left(\mathbf{A}_{m}^{4}\right)^{\top}$ is the transpose of the matrix $\mathbf{A}_{m}^{4}$. Since $\mathbf{T}_{m-1}^{N}$ is an orthogonal matrix, we have $\left(\mathbf{T}_{m-1}^{4}\right)^{\top} \overline{\mathbf{T}_{m-1}^{4}}=4 \mathbf{I}_{4}$ and

$$
\begin{aligned}
\sum_{\ell=0}^{3}\left|s_{U^{\ell}}(z)\right|^{2} & =\left(\mathbf{B}_{m-1}^{4}(z)\right)^{\top}\left(\mathbf{T}_{m-1}^{4}\right)^{\top} \overline{\mathbf{T}_{m-1}^{4} \mathbf{B}_{m-1}^{4}(z)} \\
& =4\left(\mathbf{B}_{m-1}^{4}(z)\right)^{\top} \mathbf{I}_{4} \overline{\mathbf{B}_{m-1}^{4}(z)} \\
& =4 \sum_{\ell=0}^{3}\left(\mathbf{B}_{m-1}^{\ell}(z)\right)^{\top} \overline{\mathbf{B}_{m-1}^{\ell}(z)} \\
& =4 \sum_{\ell=0}^{3}\left(\mathbf{A}_{m-1}^{\ell}(z)\right)^{\top} \overline{\mathbf{A}_{m-1}^{\ell}(z)} \\
& =\cdots \\
& =4^{m} \sum_{\ell=0}^{3}\left(\mathbf{A}_{0}^{\ell}(z)\right)^{\top} \overline{\mathbf{A}_{0}^{\ell}(z)} \\
& =4^{m+1}=4 n,
\end{aligned}
$$

where $\mathbf{I}_{4}$ is the identity $4 \times 4$ matrix. This clearly shows that $\operatorname{PMEPR}(U)=4$. By the same reason, $\operatorname{PMEPR}(V)=4$. Therefore $B=4$, which completes the proof.

\section{Code rate of $16-Q A M$ by generalized RSP}

For the generalized RSP of degree $4^{k}-1$, there are $3 k$ variables involved in $A_{k}^{0}(z)$, and each variable has 4 choices. Hence, one can construct $4^{3 k}$ QPSK sequences by generalized RSP. Since for any $\xi \in \xi^{\mathbb{Z}_{4}}, \xi a$ is a generalized RSP if $a$ is a generalized RSP, one can totally construct $4^{3 k+1}$ distinct QPSK sequences by generalized RSP. Since we choose different QPSK sequences to construct the 16-QAM OFDM signals, we have totally $\left[4^{3 k+1}\right]^{2}$ different choices. This gives the code rate of the 16-QAM by generalized RSP.

Theorem 2: For the 16-QAM codes of length $4^{k}$ by generalized RSP, the code rate is

$$
\frac{6 k+2}{4^{k}}
$$

Let $m=2 k$, the code rate is $(3 m+2) / 2^{m}$, which is higher than the constructions presented in [17], [18], [24]. Fig. 2 shows the code rate versus the code length for different constructions. One will see our performance is better than [18], [24] for the modurately large carriers. Since generalized RSP is a subset of Golay set, our curve is below that in [17]. But the generalied RSP based method in this paper is simple, efficient and practical..

\section{Hamming distance of 16-QAM by generalized RSP}

For two 16-QAM symbols $q_{1}=q\left(\eta_{1}, \zeta_{1}\right)$ and $q_{2}=$ $q\left(\eta_{2}, \zeta_{2}\right)$, we can see $q_{1} \neq q_{2}$ if either $\eta_{1} \neq \eta_{2}$ or $\zeta_{1} \neq \zeta_{2}$. Take a 16-QAM sequence $Q=\alpha e^{j \pi / 4} U+\beta e^{j \pi / 4} V$, where $U=\left(\eta_{0}, \ldots, \eta_{n-1}\right)$ and $V=\left(\zeta_{0}, \ldots, \zeta_{n-1}\right)$ are two QPSK sequences. Then the Hamming distance (HD) of $Q$ is the smaller one between the Hamming distances of $U$ and $V$. In the following theorem, the Hamming distance of 16-QAM sequences by generalized RSP is given.

Theorem 3: For the 16-QAM sequences of length $4^{k}$ by generalized RSP, the minimum Hamming distance is $4^{k-1}$.

Proof: Take the 16-QAM sequence $Q=\alpha e^{j \pi / 4} U+$ $\beta e^{j \pi / 4} V$ of length $4^{k}$. Since the hamming ditance of $Q$ is 


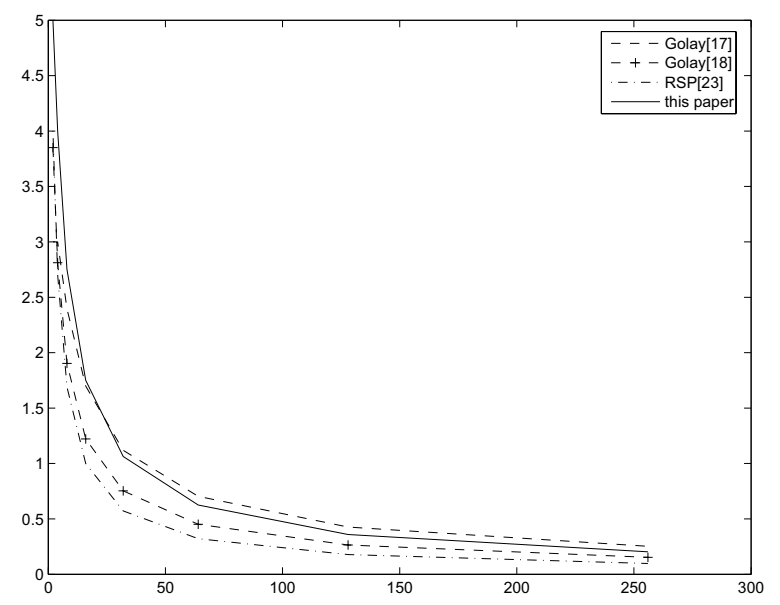

Fig. 2. The code rates of the constructions by [17], [18], [24] and this paper versus the length of the code. Our code rate is higher than those in [18], [24]. Our code rate is slightly below that in [17], but our mathod is simple, efficient and practical.

determined by those of $U$ and $V$, we just need to find out the Hamming distances of $U$ and $V$. In the following, we will use induction to show that the Hamming distances of $U$ and $V$ are $4^{k-1}$.

For $k=1$ we have $A_{1}^{0}(z)=1+\xi_{0}^{1} z+\xi_{0}^{2} z^{2}+\xi_{0}^{3} z^{3}$. For different choices of $\xi_{0}^{1}, \xi_{0}^{2}, \xi_{0}^{3}$, we obtain different codes. Then the Hamming distance of $U$ is $1=4^{k-1}$.

For the case $k=m+1$, we have

$$
A_{m+1}^{0}=A_{m}^{0}+\xi_{m}^{1} z^{4^{m}} A_{m}^{1}+\xi_{m}^{2} z^{2 \cdot 4^{m}} A_{m}^{2}+\xi_{m}^{3} z^{3 \cdot 4^{m}} A_{m}^{3}
$$

Since the degrees of $A_{m}^{0}, \cdots, A_{m}^{3}$ are $4^{m}-1$, the cofficients of $z^{0}, \cdot, z^{3 \cdot 4^{m}}$ will not add to each other. For the different choices of $\xi_{m}^{\ell}$, the derived codes are different at least at $4^{m}$ places, the length of $A_{m}^{\ell}$. Therefore the Hamming distance of the code is $4^{(m+1)-1}$. For the case of fixed choice of $\xi_{m}^{\ell}$, suppose the Hamming distance of the code by $A_{m}^{\ell}$ are $4^{m-1}$. Since each $A_{m}^{\ell}$ includes all $\xi_{i}^{\ell}$ for $\ell=0,1,2,3$ and $0 \leq i<m$, there are must one $\xi_{i}^{\ell}$ changes. This implies that all $A_{m}^{\ell}$ are not fixed choices for two different $A_{m+1}^{0}$. Therefore the code rate of $A_{m+1}^{0}$ is $4 \times 4^{m-1}=4^{(m+1)-1}$.

By induction, this proves the Hamming distance of $U$ is $4^{k-1}$. For the same reason, the Hamming distance of $V$ is also $4^{k-1}$. Therefore the Hamming distance of $Q$ is $4^{k-1}$, which completes the proof.

Let $2 k=m$, the Hamming distance is actually $2^{m-2}$ for this kind of codes of length $2^{m}$.

\section{CONCLUSIONS}

In this paper, we introduce the generalized RSP, which can recursively produce a large number of QPSK sequences. Then we use these QPSK sequencs to construct 16-QAM codewords for OFDM signals. By this construction, we can obtain $4^{3 m+2}$ 16-QAM codewordes of length $2^{m}$, while controlling the PMEPR by 7.2. Moreover, we find that the Hamming distance is $2^{m-2}$ for this code of length $2^{m}$. Since this construction is very simple and efficient, it is very practical.

\section{REFERENCES}

[1] H. Ochiai and H. Imai, "On the clipping for peak power reduction of OFDM signals," in IEEE GLOBECOM. San Francisco, USA: IEEE, 2000, pp. 731-735.

[2] W. G. Jeon, K. H. Chang, and Y. S. Cho., "An adaptive data predistorter for compensation of nonlinear distortion in OFDM systems." IEEE Trans. Commun., vol. 45, no. 10, pp. 1167-1171, Oct. 1997.

[3] J. A. Davis and J. Jedwab, "Peak-to-mean power control and error correction for OFDM transmission using Golay sequences and ReedMuller codes," IEE Elect. Lett., vol. 33, no. 4, pp. 267-268, Feb. 1997.

[4] —, "Peak-to-mean power control in OFDM, Golay complementary sequences, and Reed-Muller codes," IEEE Trans. Inform. Theory, vol. 45, no. 7, pp. 2397-2417, Nov. 1999.

[5] K. G. Paterson and V. Tarokh, "On the existence and construction of good codes with low peak-to-average power ratio," IEEE Trans. Inform. Theory, vol. 46, no. 6, pp. 1974-1987, Sept. 2000.

[6] K. G. Paterson, "Generalized Reed-Muller codes and power control in OFDM modulation," IEEE Trans. Inform. Theory, vol. 46, no. 1, pp. 104-120, Jan. 2000.

[7] H. Breiling, S. H. Muller-Weinfurtner, and J. B. Huber, "SLM peakpower reduction without explicit side information," IEEE Commun. Lett., vol. 5, no. 6, pp. 239-241, 2001.

[8] R. W. Bauml, R. F. H. Fischer, and J. B. Huber, "Reducing the peak-toaverage power ratio of multicarrier modulation by selected mapping,' IEE Elect. Lett., vol. 32, no. 22, pp. 2056-2057, Oct. 1996.

[9] G. Hill, M. Faulkner, and J. Singh, "Cyclic shifting and time inversion of partial transmit sequences to reduce the peak-to-average power ratio in OFDM," in IEEE PIMRC, vol. 2. Piscataway, NJ, USA.: IEEE, 2000, pp. 1256-1259, conference Paper.

[10] P. V. Eetvelt, G. Wade, and M. Thompson, "Peak to average power reduction for OFDM schemes by selected scrambling," IEE Elect. Lett., vol. 32, no. 21, pp. 1963-1964, Oct. 1996.

[11] P. K. Frenger and N. A. B. Sevensson, "Parallel combinatory OFDM signalling," IEEE Trans. Commun., vol. 47, no. 4, pp. 558-567, Apr. 1999.

[12] J. Tellado and J. M. coiffi, "PAR reduction in multicarrier transmission systems," Stanford University," Technical Report, 1998.

[13] S. Boyd, "Multitone signals with low crest factors," IEEE Trans. Circuits Syst., vol. CAS-33, no. 10, pp. 1018-1022, Oct 1986.

[14] B. M. Popovic, "Synthesis of power efficient multitone signals with flat amplitude spectrum," IEEE Trans. Commun., vol. 39, pp. 1031-1033, July 1991.

[15] M. J. E. Golay, “Complementary series," IRE. Trans. Inform. Theory, vol. IT-7, pp. 82-87, Apr. 1961.

[16] C. Tseng and C. Liu, "Complementary sets of sequences," IEEE Trans. Inform. Theory., vol. 18, no. 5, pp. 644-652, Sept. 1972.

[17] C. Röbing and V. Tarokh, "A construction of ofdm 16-qam sequences having low peak powers,"," IEEE Trans. Inform. Theory., vol. 47, no. 11, pp. 2091-2094, Nov. 2001.

[18] R. V. C. V. Chong and V. Tarokh, "A new consruction of 16-qam golay complementary sequences," IEEE Trans. Inform. Theory., vol. 49, no. 11, pp. 2953-2959, Nov. 2003.

[19] W. Rudin, "Some theorems on Fourier coefficients," Proc. Amer. Math. Soc., vol. 10, pp. 855-859, 1959

[20] W. Chen and C. Tellambura, "A good trade-off performance between the code rate and the pmepr for ofdm signals using the generalized rudinshapiro polynomials," in IEEE ICC. Seoul, Korea: IEEE, 2005, pp. 2600-2604.

[21] J. Zhao, "A peak-to-mean power control scheme: the extended rudinshapiro construction," in VTC, vol. 1. Los Angeles, USA: IEEE, 2004, pp. 373-377.

[22] _ - "Efficient identification of the codewords with low peak-to-mean envelope power ratio of multicarrier transmission," in VTC, vol. 1. Los Angeles, USA: IEEE, 2004, pp. 621-625.

[23] A. J. Grant and R. Van Nee, "Efficient maximum likelihood decoding of peak power limiting codes for OFDM," in IEEE Vehicular Technology Conference. IEEE, 1998, pp. 2081-2084.

[24] J. Zhao, "A new construction of 16-qam codewords with low peak power," in ICC. IEEE, 2006.

[25] A. J. Grant and R. Van Nee, "Efficient maximum-likelihood decoding of Q-ary modulated Reed-Muller codes," IEEE Commun. Lett., vol. 2, no. 5 , pp. 134-136, may 1998 . 